\section{EGF-CFC proteins are essential coreceptors for the TGF- $\beta$ signals Vg1 and GDF1}

\author{
Simon K. Cheng, ${ }^{1}$ Felix Olale, ${ }^{1}$ James T. Bennett, ${ }^{1}$ \\ Ali H. Brivanlou, ${ }^{2,4}$ and Alexander F. Schier ${ }^{1,3}$ \\ ${ }^{1}$ Developmental Genetics Program, Skirball Institute of \\ Biomolecular Medicine, Department of Cell Biology, New \\ York University School of Medicine, New York, New York \\ 10016, USA; ${ }^{2}$ Laboratory of Molecular Vertebrate Embryology, \\ The Rockefeller University, New York, New York 10021, USA
}

The TGF- $\beta$ signals Nodal, Activin, GDF1, and Vg1 have been implicated in mesoderm induction and left-right patterning. Nodal and Activin both activate Activin receptors, but only Nodal requires EGF-CFC coreceptors for signaling. We report that Vg1 and GDF1 signaling in zebrafish also depends on EGF-CFC proteins, but not on Nodal signals. Correspondingly, we find that in Xenopus Vg1 and GDF1 bind to and signal through Activin receptors only in the presence of EGF-CFC proteins. These results establish that multiple TGF- $\beta$ signals converge on Activin receptor/EGF-CFC complexes and suggest a more widespread requirement for coreceptors in TGF- $\beta$ signaling than anticipated previously.

Received September 12, 2002; revised version accepted November 8, 2002.

EGF-CFC proteins are membrane-bound extracellular factors with essential roles during vertebrate development. Members of the EGF-CFC family consist of Oneeyed pinhead (Oep) in zebrafish, FRL1 in Xenopus, Cryptic in chick, and Cripto and Cryptic in mouse and human (for review, see Shen and Schier 2000). Genetic studies in zebrafish and mouse have shown that EGF-CFC proteins are required for mesoderm and endoderm induction and left-right axis formation. Zebrafish embryos lacking both the maternal and zygotic contribution of Oep (MZoep) are defective in mesendoderm induction (Gritsman et al. 1999). Similarly, mouse cripto mutants fail to form a primitive streak and lack embryonic mesoderm (Ding et al. 1998). During later stages of development, EGF-CFC genes are required for proper left-right axis formation. Loss of late Oep activity results in embryos that lack expression of left-side-specific genes and display randomization of left-right laterality (Yan et al. 1999). Similarly, mouse cryptic mutants do not express left-sidespecific genes and have left-right defects such as heterotaxia and right isomerism (Gaio et al. 1999; Yan et al. 1999). In addition, mutations in cryptic/CFC1 are asso-

[Keywords: TGF- $\beta$; EGF-CFC; Nodal; Activin receptors; mesoderm induction; left-right axis]

Corresponding authors.

${ }^{3}$ E-MAIL schier@saturn.med.nyu.edu; FAX (212) 263-7760.

${ }^{4}$ E-MAIL brvnlou@rockvax.rockefeller.edu; FAX (212) 327-8685.

Article and publication are at http://www.genesdev.org/cgi/doi/10.1101/ gad.1041203. ciated with laterality defects in humans (Bamford et al. 2000).

Genetic studies have shown that EGF-CFC proteins are essential for signaling by TGF- $\beta$ signals of the Nodal family (Gritsman et al. 1999). Double mutants for the zebrafish nodal-related genes cyclops and squint are phenotypically identical to MZoep mutants (Feldman et al. 1998; Gritsman et al. 1999). Moreover, Nodal signals are inactive in MZoep mutants (Gritsman et al. 1999). Biochemically, the EGF-CFC protein Cripto can act as a coreceptor for Nodal signaling (Reissmann et al. 2001; Yeo and Whitman 2001; Bianco et al. 2002; Sakuma et al. 2002; Yan et al. 2002). Current evidence suggests that Cripto binds to the Activin type I receptor Alk4 and forms a complex with Nodal and the type II Activin receptor ActRIIB (for review, see Whitman 2001). Upon receptor activation, the intracellular kinase domain of the type I receptor phosphorylates the signal transducers Smad2 and/or Smad3 (for review, see Massague and Chen 2000). During mesoderm induction, this leads to the expression of downstream genes such as goosecoid and brachyury/T/no tail (for review, see Schier and Shen 2000; Whitman 2001). Further support for an essential role of EGF-CFC proteins in Nodal signaling is provided by the observations that some hypomorphic or conditional mouse nodal mutants display left-right defects resembling cryptic mutants (Lowe et al. 2001; Brennan et al. 2002; Norris et al. 2002), and strong nodal mutants share aspects of the cripto phenotype (Conlon et al. 1994; Lowe et al. 2001; Norris et al. 2002).

In light of analyses of other TGF- $\beta$ signals, the requirement for EGF-CFC proteins as Nodal coreceptors has appeared unusual (Massague and Chen 2000). With the exception of the TGF- $\beta$ type III receptor in TGF- $\beta 2$ signaling, and of endoglin in Alk1-mediated signaling, no coreceptors have been implicated in TGF- $\beta$ signaling (Massague and Chen 2000). The use of EGF-CFC coreceptors also seems uncommon because genetic and biochemical studies have shown that Activin utilizes the same receptors, Alk4 and ActRIIB, as Nodal but does not require EGF-CFC coreceptors (Massague and Chen 2000; Schier and Shen 2000; Whitman 2001). In contrast to Nodal, Activin can activate downstream signaling and induce mesoderm formation in both wild-type and MZoep mutant embryos (Gritsman et al. 1999). Moreover, Activin can bind to Alk4 and ActRIIB in the absence of EGF-CFC proteins (Massague and Chen 2000). It has thus been unclear whether the Nodal/EGF-CFC interaction is unusual or whether other TGF- $\beta$ signals rely on coreceptors such as EGF-CFC.

A third class of TGF- $\beta$ ligands exhibits similar biological activities as members of the Nodal and Activin families. Signals belonging to the Vg1/GDF1 family (Vg1 in Xenopus, zebrafish and chick; GDF1 in mouse) share only $35 \%-55 \%$ identity with Nodal or Activin in the mature domain, but can also act as mesoderm inducers (Schier and Shen 2000; Whitman 2001). Processed bVg1 and bGDF1 (chimeras between the $\mathrm{N}$-terminal prodomain of BMP and the C-terminal mature domains of $\mathrm{Vg} 1$ and GDF1) induce mesodermal markers in Xenopus (Thomsen and Melton 1993; Kessler and Melton 1995; Wall et al. 2000). Similarly, grafts of cells expressing native $\mathrm{cVg} 1$ or chimeric BMP-cVg1 initiate formation of 
ectopic primitive streaks in chick (Seleiro et al. 1996; Skromne and Stern 2002). It has been proposed that Vg1 acts upstream of Nodal signals in this process (Wall et al. 2000; Skromne and Stern 2002). Xenopus Vg1 is expressed maternally before the transcription of nodal genes (Weeks and Melton 1987) and misexpression of bVg1 induces ectopic Xnr1 (Xenopus nodal related 1) expression (Hyatt et al. 1996; Hyatt and Yost 1998; Wall et al. 2000). Similarly, cVg1 is expressed before nodal during chick embryogenesis and misexpression of $\mathrm{cVg} 1$ in the anterior marginal zone during gastrulation induces ectopic nodal expression (Skromne and Stern 2002). GDF1 and Vg1 have also been implicated upstream of Nodal signals during left-right axis formation. GDF1 mouse mutants have left-right laterality defects and lack nodal expression in the left lateral plate (Rankin et al. 2000). Moreover, bVg1 can induce leftness in Xenopus upstream of Nodal signals (Hyatt et al. 1996; Hyatt and Yost 1998; Ramsdell and Yost 1999).

Despite the pivotal roles of Vg1/GDF1 during embryogenesis, the signaling pathway activated by these TGF- $\beta$ signals has not been defined molecularly. Several lines of evidence suggest that Vg1 and GDF1 may activate the same or related pathways as Activin and Nodal. First, Vg1 signaling results in the phosphorylation of Smad2 (Lee et al. 2001). Second, mesoderm induction by bGDF1 and bVg1 can be inhibited by the Smad2-interaction domain (SID) of FAST1, which blocks formation of Smad2Smad4 complexes (Wall et al. 2000). Third, a truncated form of ActRIIB (DXAR1; Hemmati-Brivanlou and Melton 1992) missing the cytoplasmic kinase domain, can block Vg1 and GDF1 signaling (Kessler and Melton 1995; Wall et al. 2000). However, direct binding of Vg1 to ActRIIB has not been detected (Kessler and Melton 1995). Here, we present genetic and biochemical studies in zebrafish and Xenopus that indicate that $\mathrm{Vgl}$ and GDF1 act similarly to Nodal and depend on EGF-CFC coreceptors for the interaction with and activation of Activin receptors.

\section{Results and Discussion}

\section{Vg1 and GDF1 signaling requires zebrafish One-eyed pinhead}

To determine the requirement of EGF-CFC genes in $\mathrm{Vg} 1$ and GDF1 signaling, we tested the activity of these TGF- $\beta$ signals in zebrafish one-eyed pinhead mutants, which lack EGF-CFC activity. To facilitate efficient processing and generation of active ligands (Thomsen and Melton 1993; Wall et al. 2000), we used the chimeric constructs aVg1 (Activin $\beta \mathrm{B}$ prodomain fused to mature domain of zebrafishVg1) and bGDF1 (BMP4 prodomain fused to the mature domain of mouse GDF1; Wall et al. 2000). To test whether Vg1 and GDF1 signaling requires Oep, we injected mRNAs encoding aVgl or bGDF1 into wild-type or MZoep embryos. As a readout for active signaling, we analyzed the ectopic induction of the mesodermal marker no tail (ntl) and the organizer marker goosecoid $(g s c)$. As observed previously (Gritsman et al. 1999|, control injections of mRNA encoding the Nodal signal Squint induced $n t l$ and $g s c$ in wild-type embryos (Figs. 1P, 2P), but not in MZoep mutants (Figs. 1Q, 2Q). In contrast, Activin induced $n t l$ and gsc expression in both wild-type and MZoep embryos (Fig. 1S,T; Gritsman

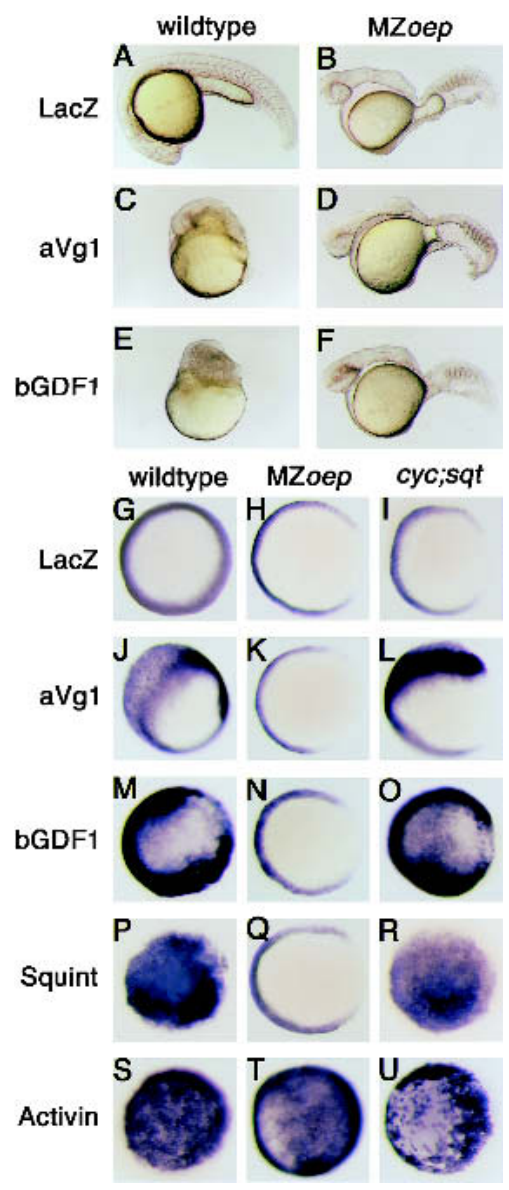

Figure 1. Dependence of $\mathrm{Vg} 1$ and GDF1 signaling on Oep but not on Nodal signals. $(A-F)$ Live zebrafish embryos at $24 \mathrm{hpf}$, anterior to the left, dorsal up. $(G-U) n t l$ mRNA expression in zebrafish embryos at shield stage, animal pole view; dorsal to the right in $H, I, I, K, L$, $N, O$, and $Q$. $(A, G)$ LacZ control-injected wild type. $(B, H) L a c Z$ control-injected MZoep. $(A, C, E)$ Wild-type embryos. $(G, J, M, P, S)$ Wild-type heterozygous siblings $\left(\mathrm{cyc}^{\mathrm{m} 294} /+; s q^{c z 35} /+\right.$ from a $c y c^{m 294} /+$; $s q^{t c z 35} /+$ intercross). $(B, D, F, H, K, N, Q, T)$ MZoep embryos. $(I, L, O, R, U)$ cyc;sqt double mutant embryos. Embryos were injected with $250 \mathrm{pg}$ of LacZ mRNA $(A, B, G, H, I) ; 100 \mathrm{pg}(C, J, K, L)$ or $250 \mathrm{pg}(D)$ of $a V g 1$ mRNA; $100 \mathrm{pg}(E)$ or $250 \mathrm{pg}(F, M, N, O)$ of $b G D F 1$ mRNA; $20 \mathrm{pg}$ of squint mRNA $(P, Q, R)$; and $20 \mathrm{pg}(T)$ or $100 \mathrm{pg}(S, U)$ of activin mRNA. $(G)$ Note the wild-type expression of $n t l$ in marginal cells. $(H)$ In MZoep mutants, ntl expression is absent dorsally at the margin. Wild-type embryos are severely dorsalized or have ectopic $n t 1$ expression when injected with $a V g 1(C, J), b G D F 1(E, M)$, squint $(P)$, and activin $(S)$ mRNA. In contrast, MZoep embryos injected with $a V g 1(D, K), b G D F 1(F, N)$, or squint $(Q)$ mRNA are indistinguishable from the LacZ control-injected embryos. Similar to wild-type embryos, expression of $n t l$ is expanded in $c y c$; sqt double mutants injected with $a V g 1(L), b G D F 1(O)$, squint $(R)$, or activin $(U)$ mRNA.

et al. 1999). Misexpression of aVg1 and bGDF1 in wildtype embryos resulted in severe dorsalization (Fig. 1C,E) and induced the ectopic expression of $n t l$ (Fig. 1J,M) and gsc (Fig. 2F,K). In contrast, injections into MZoep mutants did not lead to any phenotypic changes (Fig. 1D,F) and failed to induce ectopic $n t l$ (Fig. $1 \mathrm{~K}, \mathrm{~N}$ ) or gsc expression (Fig. 2G,L). Similar to Vg1 and GDF1, the TGF- $\beta$ signal Derrière induces Smad2 phosphorylation in Xenopus (Lee et al. 2001) and induces gsc and $n t l$ in wild-type zebrafish embryos (data not shown). In contrast to GDF1 and $\mathrm{Vg} 1$, Derrière can induce $n t l$ expression and trunk formation in MZoep mutants, but it only weakly in- 


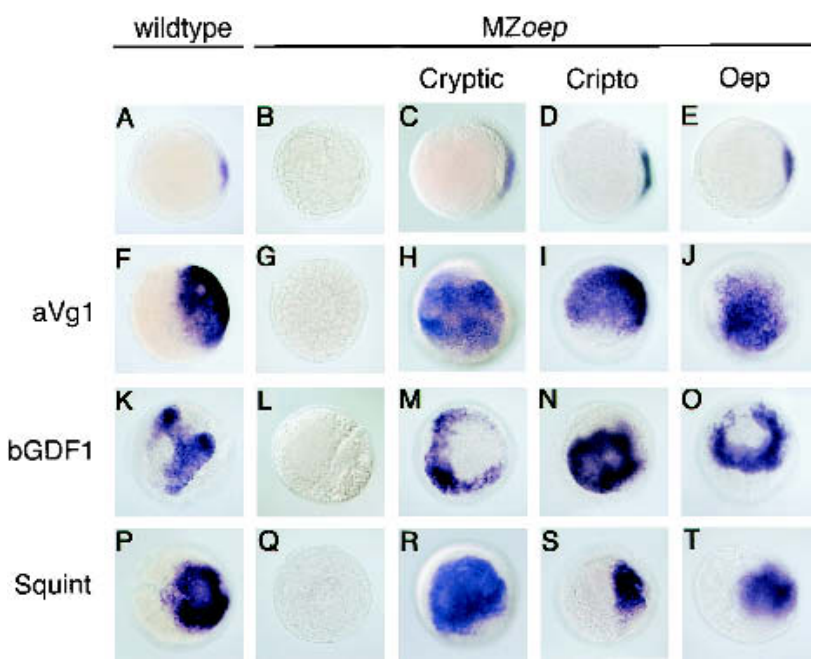

Figure 2. $\mathrm{Vgl}$ and GDF1 signaling is restored in MZoep embryos by divergent EGF-CFC proteins. $(A-T) g s c$ mRNA expression in zebrafish embryos at shield stage, animal pole view; dorsal to the right in $A, C, D, E, F, P, S$, and $T)$. $(A, F, K, P)$ Wild-type embryos. $(B--E, G-$ $J, L--O, Q--T)$ MZoep embryos. (A) Uninjected wild type. (B) Uninjected MZoep. Note the wild-type expression of gsc in the dorsal organizer. In MZoep mutants, gsc expression is absent. Wild-type embryos $(F, K, P)$ and MZoep mutants $(G, L, Q)$ injected with $100 \mathrm{pg}$ of $a V g 1(F, G), 250 \mathrm{pg}$ of $b G D F 1(K, L)$, and $100 \mathrm{pg}$ of squint $(P, Q)$ mRNA. $g s c$ is ectopically expressed when $a V g 1(F), b G D F 1(K)$, or squint $(P)$ mRNA are injected in wild-type embryos, but not in MZoep embryos $(G, L, Q$, respectively). MZoep embryos injected with $50 \mathrm{pg}$ each of cryptic $(C)$, cripto $(D)$, or oep $(E)$ mRNA. Note the rescue of the dorsal organizer expression of gsc in injected MZoep. One-hundred picograms of $a V g 1$ mRNA was coinjected into MZoep with $50 \mathrm{pg}$ each of cryptic $(H)$, cripto $(I)$, or oep $(J)$ mRNA. Twohundred-fifty picograms of $b G D F 1$ mRNA was coinjected into MZoep with $50 \mathrm{pg}$ each of cryptic $(M)$, cripto $(N)$, or oep $(O)$ mRNA. One-hundred picograms of squint mRNA was coinjected into MZoep with $50 \mathrm{pg}$ each of cryptic $(R)$, cripto $(S)$, or oep $(T)$ mRNA. Coinjection of either cryptic, cripto, or oep mRNA with aVg1 $(H, I, J$, respectively), bGDF1 ( $M, N, O$, respectively), or squint $(R, S, J$, respectively) mRNA results in ectopic gsc expression in MZoep.

duces gsc expression (data not shown). These results establish that, in contrast to Activin, but similar to Nodal signaling, aVg1 and bGDF1 signaling is dependent on Oep.

\section{Vg1 and GDF1 signaling is independent of zebrafish} Nodal signals

Although the above results show that Vg1 and GDF1 signaling depends on Oep, it is possible that this requirement is indirect, because Vg1 and GDF1 might act upstream of or in parallel to Nodal signals (Hyatt et al. 1996; Hyatt and Yost 1998; Rankin et al. 2000; Wall et al. 2000; Skromne and Stern 2002). In this scenario, Vg1 and GDF1 signaling would be dependent on Nodal signals, which, in turn, are dependent on Oep. To test the epistastic relationship between Nodal and Vg1 or GDF1, we injected mRNAs encoding Activin, Squint, aVg1, or bGDF1 into double mutants for the zebrafish Nodal signals cyclops and squint. All four TGF- $\beta$ signals induced ectopic $n t l$ expression in cyc;sqt double mutants (Fig. $1 \mathrm{~L}, \mathrm{O}, \mathrm{R}, \mathrm{U})$. These results establish that bGDF1 and aVg1 signaling is independent of the Nodal signals Cyclops and Squint but requires the EGF-CFC protein Oep.

\section{Mouse Cryptic and Cripto can mediate Vg1 and GDF1 signaling}

Previous studies have shown that expression of the heterologous EGF-CFC proteins Cripto and Cryptic in MZoep mutants can restore Nodal signaling (Gritsman et al. 1999; Bamford et al. 2000). In the case of gsc, injection of cryptic, cripto, or oep mRNA into MZoep mutants rescues organizer expression (Fig. 2C-E; Gritsman et al. 1999). Coinjection of squint mRNA induces ectopic expression of gsc (Fig. 2R-T). To test whether Vg1 and GDF1 signaling can also be mediated by different EGF-CFC proteins, we coinjected mRNAs encoding aVg1 or bGDF1 into MZoep embryos expressing Oep, mouse Cryptic, or mouse Cripto. In all combinations, ectopic expression of gsc was induced (Fig. 2H-J,M-O). These results establish that Vg1 and GDF1 signaling can be mediated by divergent EGF-CFC proteins.

\section{Vg1 and GDF1 signal through ActRIIB, Alk4, and EGF-CFC proteins}

To determine the receptor specificity of Vg1 and GDF1, we used a chimeric receptor assay in Xenopus embryos. Because Activin and Nodal can both activate the type I Activin receptor Alk4, we tested whether Vg1 or GDF1 can also signal through Alk4. To separate the effect of Vg1 and GDF1 on Alk4 from the activation of endogenous receptors, we generated a chimeric receptor Alk4/ $3 \mathrm{C}$. This chimera contains the extracellular and transmembrane domains of Xenopus Alk4 and the intracellular kinase domain of the type I BMP receptor, Xenopus Alk3 (also known as BMPRIA). We reasoned that this chimera binds to its ligands, but instead of activating the Smad2/3 pathway, will activate the Smad1/5 pathway (Yeo and Whitman 2001). For the type II receptor, we used a nonchimeric type II receptor, Xenopus ActRIIB (also known as XAR1), because a truncated form of ActRIIB (Hemmati-Brivanlou and Melton 1992) can block Vg1 and GDF1 signaling (Kessler and Melton 1995; Wall et al. 2000). Moreover, ActRIIB can recruit both Alk4 for activin-like signaling and Alk3 for BMP-like signaling (Massague and Chen 2000). We used a luciferase reporter, BRE(-243/-191)x4-luc, containing a BMP response element from the $x$ Vent 2 promoter, as the readout for receptor activation (Hata et al. 2000). To block endogenous BMP signaling, we coexpressed the BMP inhibitor, Noggin (Zimmerman et al. 1996). This heterologous system has been used previously to successfully reconstitute the interactions of mouse Nodal and Cripto with Activin receptors (Yeo and Whitman 2001). Using this assay, we find that, similar to Nodal signals, aVg1 and bGDF1 can efficiently signal through ActRIIB and Alk4/3C in the presence, but not absence of Cripto (Fig. 3). These results suggest that Nodal, GDF1, and Vg1 signal through ActRIIB, Alk4, and EGF-CFC proteins.

\section{Vg1 and GDF1 bind to ActRIIB/Alk4/EGF-CFC receptor complexes}

Because Vg1 and GDF1 can signal through ActRIIB and Alk4, we determined whether these TGF- $\beta$ signals act like Nodal and bind to ActRIIB/Alk4/EGF-CFC complexes (Reissmann et al. 2001; Yeo and Whitman 2001; Bianco et al. 2002; Yan et al. 2002). We coinjected mRNA 


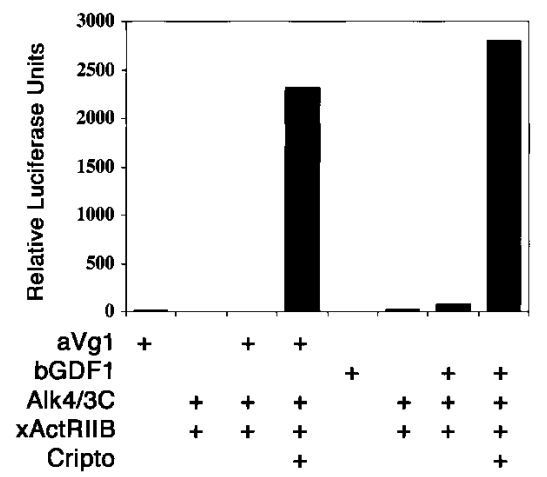

Figure 3. Vg1 and GDF1 signal through the Activin receptors, ActRIIB and Alk4, and Cripto. Synthetic mRNAs encoding aVg1 (50 pg), bGDF1 (50 pg), Alk4/3C (12.5 pg), xActRIIB (12.5 pg), and Cripto (50 pg) were injected into Xenopus embryos. Activation of the chimeric receptor was measured by a BMP response element luciferase reporter, BRE(-243/-191)x4-luc. Results are shown from one experiment and are representative of three independent experiments. Note that Alk4/3C and xActRIIB only are not responsive to $\mathrm{aVg} 1$ and bGDF1 ligands. In the presence of Cripto, aVg1 and bGDF1 signaling through ActRIIB and Alk4/3C is greatly enhanced. Low levels of activation can sometimes be seen when coexpressing the ligand and receptors only. This is presumably due to the endogenous expression of EGF-CFC proteins, such as FRL1, in these embryos.

encoding epitope-tagged ligands (aVg1/HA or bGDF1/ HA), receptors (xActRIIB/Myc and xAlk4, or mActRIIB (KR)/Myc and hAlk4(KR)/Flag) and coreceptors (Cripto/ Flag or Cryptic/Flag) into Xenopus embryos (Yeo and Whitman 2001). After chemical cross-linking to covalently stabilize protein complexes, coimmunoprecipitations against epitope-tagged proteins were performed. Similar to Nodal (Reissmann et al. 2001; Yeo and Whitman 2001), mature Vg1 and GDF1 ligands, when coexpressed with ActRIIB and Alk4, can bind to ActRIIB only in the presence of the EGF-CFC proteins Cripto and Cryptic, respectively (Fig. 4A,B). We examined further whether Vg1 or GDF1 can, similar to Nodal (Reissmann et al. 2001; Bianco et al. 2002; Yan et al. 2002), interact with EGF-CFC proteins without coexpressing the receptors. In support of this hypothesis, Cripto coimmunoprecipitated with aVg1 and Cryptic coimmunoprecipitated with bGDF1 (Fig. 4C). These results indicate that similar to Nodal, Vg1 and GDF1 bind to ActRIIB/Alk4/EGF-CFC receptor complexes.

The molecular basis of $\mathrm{Vg} 1$ and GDF1 signaling has been elusive. Our genetic and biochemical studies in zebrafish and Xenopus now indicate that Vg1 and GDF1 depend on EGF-CFC coreceptors for the interaction with and activation of Activin receptors. These results have important implications for the molecular and developmental control of TGF- $\beta$ signaling. First, our findings establish that multiple TGF- $\beta$ signals (Nodal, Vg1, and GDF1) converge on Activin receptor/EGF-CFC complexes. This suggests that the dependence of Nodal signaling on EGF-CFC coreceptors is not an unusual case, but might reveal a more widespread requirement for coreceptors in TGF- $\beta$ signaling than anticipated previously. It remains to be determined whether the dependence of Nodal, GDF1, and Vg1 on EGF-CFC coreceptors is universal, or whether there are ligand or receptor combinations in systems other than the zebrafish embryo that can be activated by these TGF- $\beta$ signals in the absence of EGF-CFC proteins. Second, the finding that Activin activates the same receptors as Nodal and $\mathrm{Vgl} /$ GDF1 independently of EGF-CFC proteins raises the question as to which sequences confer independence from EGF-CFC proteins. Sequence comparisons indicate that highest divergence between Activin, Nodal, and $\mathrm{Vg} 1 / \mathrm{GDF} 1$ are in the $\mathrm{N}$-terminal segment of finger 1 , the central $\alpha$-helix, and the turn of the finger 2 regions. These regions are potential candidates to determine the

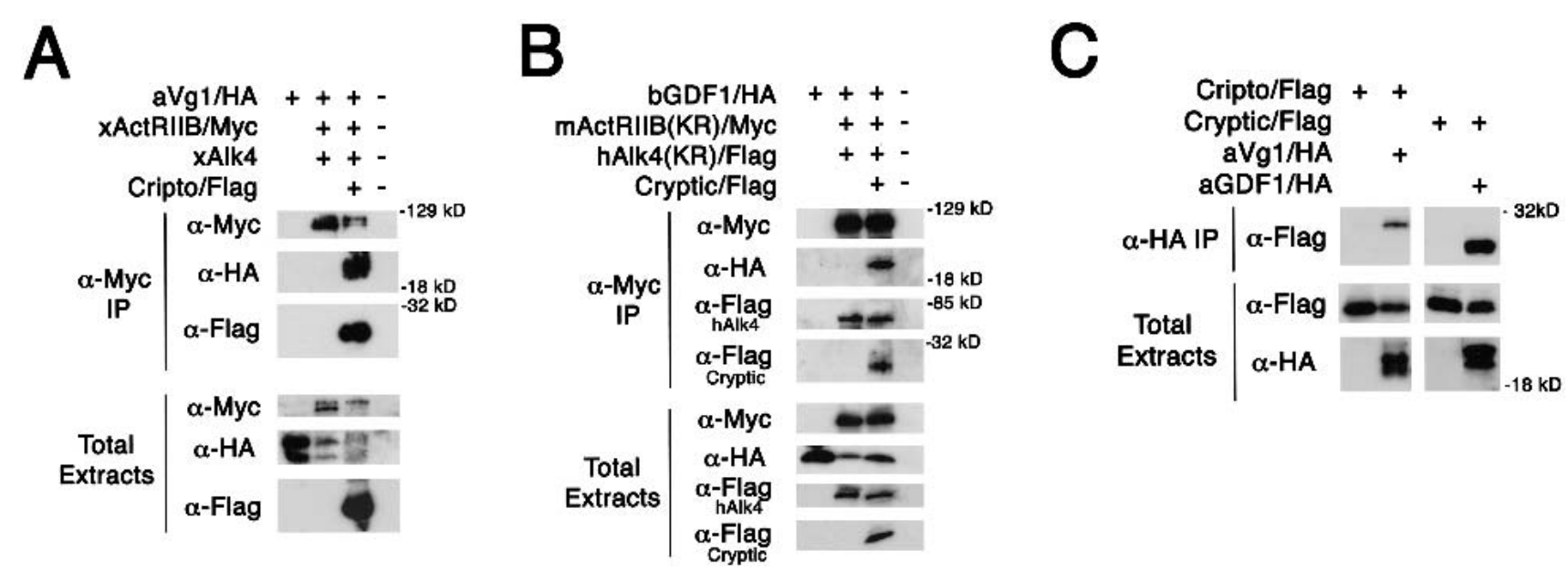

Figure 4. Vg1 and GDF1 binding to the ActRIIB and Alk4 receptor complex requires EGF-CFC proteins. aVg1 $(A)$ and bGDF1 $(B)$ binding to receptor complex. RNAs encoding aVg1/HA (100 pg), xActRIIB/Myc (500 pg), xAlk4 (500 pg), Cripto/Flag (1 ng), bGDF1/HA (2 ng), mActRIIB (KR)/Myc (1 ng), hAlk4(KR)/Flag (1 ng), and Cryptic/Flag (1 ng) were injected into Xenopus embryos. After chemical cross-linking with DTSSP, lysates were immunoprecipitated for either xActRIIB/Myc $(A)$ or mActRIIB(KR)/Myc $(B)$ with $\alpha$-Myc antibody. Note that Cripto and Cryptic form a complex with the type II and type I receptors and the mature ligands Vg1 and GDF1, respectively. (C) Vg1 and GDF1 interact with EGF-CFC proteins. Synthetic mRNAs encoding Cripto/Flag (1 ng), Cryptic/Flag (1 ng), aVg1/HA (1 ng), and aGDF1/HA (1 ng) were injected into Xenopus embryos. After DTSSP cross-linking, lysates were immunoprecipitated for either aVg1/HA or aGDF1/HA with $\alpha$-HA antibody. Proteins in the coimmunoprecipitates and total extracts were probed in Western blot analysis with the indicated antibodies: xActRIIB/Myc $(\sim 120 \mathrm{kD} ; \alpha-\mathrm{Myc})$, mActRIIB(KR)/Myc ( $120 \mathrm{kD}, \alpha-\mathrm{Myc})$, aVg1/HA ( 22 kD; $\alpha$-HA), bGDF1/HA ( 22 kD, $\alpha-\mathrm{HA}), \mathrm{aGDF} 1 / \mathrm{HA}(\sim 22 \mathrm{kD}, \alpha-\mathrm{HA})$, hAlk4(KR)/Flag ( 70 kD; $\alpha$-Flag), Cripto/Flag ( 30 kD; $\alpha$-Flag), and Cryptic/Flag ( 25 kD; $\alpha$-Flag). 
specificity of receptor/coreceptor/ligand interactions. Third, Cripto up-regulation has been implicated in tumor formation, particularly in breast cancer (Salomon et al. 2000). Because Nodal is only expressed at very low levels in mammary tissue (Bianco et al. 2002), our results raise the possibility that Cripto mediates the signaling of another, potentially Vg1/GDF1-like, TGF- $\beta$ signal during cancer formation. Fourth, the finding that not only Nodal, but also Vg1/GDF1 signals depend on EGF-CFC proteins, leads to a novel view of mutational analyses of these genes during development. Our results suggest that EGF-CFC mutations result not only in a block of Nodal but also Vg1/GDF1 signaling. In particular, the cryptic phenotype has been interpreted as a defect in Nodal signaling during left-right patterning (Gaio et al. 1999; Yan et al. 1999; Bamford et al. 2000), whereas GDF1 was thought to act upstream of Nodal signaling (Rankin et al. 2000; Wall et al. 2000). We now show that GDF1 requires EGF-CFC proteins for signaling and can form a complex with Cryptic. Moreover, the GDF1 and cryptic mutant phenotypes are essentially identical (Gaio et al. 1999; Yan et al. 1999; Rankin et al. 2000). We thus propose that GDF1 and Nodal cooperate and both converge on Cryptic to activate left-side-specific gene expression. Similarly, our results predict that the loss of all EGF-CFC genes leads to the same or similar phenotype as the combined loss of Vg1/GDF1 and nodal genes. In the mouse, this model predicts that GDF1;nodal double mutants have the same or similar phenotype as cripto;cryptic double mutants. Intriguingly, GDF1 and nodal have overlapping expression domains throughout embryogenesis, including the embryonic ectoderm, primitive node, and lateral plate mesoderm (Conlon et al. 1994; Rankin et al. 2000). Similarly, the expression of cripto and cryptic temporally overlaps during early mouse development. (Shen et al. 1997; Ding et al. 1998). It is thus possible that GDF1 and Cryptic have earlier roles that overlap with the ones of Nodal and Cripto, respectively. Our results lead us to speculate that GDF1;nodal double mutants will resemble cripto;cryptic double mutants and will uncover additional roles for these TGF- $\beta$ signals and their coreceptors during vertebrate embryogenesis.

\section{Materials and methods}

Strains and embryos

Generation of adult homozygous fish for $\operatorname{oep}^{\mathrm{tz} 57}$ and phenotypic analysis has been performed as described (Zhang et al. 1998; Gritsman et al. 1999). Genotyping of $c y c^{\mathrm{m} 294} ; s q t^{\mathrm{cz} 35}$ mutants was performed as described (Feldman et al. 1998; Sampath et al. 1998).

\section{Generation of constructs}

Epitope-tagged and chimeric constructs were made using PCR-based methods and confirmed by sequencing. pcDNA-aVg1/HA, pcDNAaGDF1/HA, and pcDNA-aSquint were generated by subcloning the prodomain of Xenopus Activin $\beta B$ (codons Met 1-Gly 256) fused to a HA-epitope/Xhol fragment (YPYDVPDYALE) and followed by the mature ligand domains of zebrafish Vg1 (codons Lys 239-Arg 355), mouse GDF1 (codons His 238-Arg 357) and zebrafish Squint (codons Asn 263His 392 1, respectively, into pcDNA3. pCS-bGDF1/HA was generated by inserting the HA-epitope after Leu 289 in pCS2-BMP2-GDF1 (Wall et al. 2000). pCS-hAlk4(KR)/Flag was generated by subcloning the coding region of pCS-hAlk4(KR)/HA (a kinase-defective mutant of human Alk4 with Lys 234 to Arg 234 substitution) into pCS4+3Flag vector (Yeo and
Whitman 2001). pCS-mActRIIB(KR)/Myc contains a kinase-defective mutant of mouse ActRIIB with Lys 217 to Arg 217 substitution and the Myc-epitope (Yeo and Whitman 2001). pCS-Cryptic/Flag was generated by subcloning the Cryptic coding region from $\mathrm{pT}_{7} \mathrm{~T}_{\mathrm{S}}$-Cryptic and inserting the Flag-epitope after codon Ser 25. $\mathrm{pT}_{7} \mathrm{~T}_{\mathrm{S}}-\mathrm{Alk} 4 / 3 \mathrm{C}$ was generated by fusing codons Met 1 to Glu 120 of Xenopus Alk4 and codons Phe 149 to Ile 527 of Xenopus Alk3.

\section{Embryo microinjection}

Plasmids were linearized and sense strand-capped mRNA was synthesized using the mMESSAGE mMACHINE system (Ambion). Zebrafish embryos were dechorinated by pronase treatment and injected between the 1- and 4-cell stage. Xenopus embryos at the 1-2-cell stage were used for injections into the animal pole.

\section{Luciferase reporter assay}

Luciferase assays were performed in triplicate with three embryos each. The injection mixtures were equalized with respect to total mRNA amount with LacZ mRNA. noggin (100 pg) mRNA and BRE(-243/191) x4-luc DNA construct (25 pg; Hata et al. 2000) were included in each sample. Whole Xenopus embryos were harvested at stage 9. Luciferase activity was analyzed using the Luciferase Reporter Assay system (Promega) according to the manufacturer's instruction in a Lumat LB9501 (Berthold Technologies).

\section{Coimmunoprecipitation analysis}

Xenopus embryos were harvested at stage 10 . For chemical cross-linking of proteins, animal halves were incubated in PBS with $10 \mathrm{mM}$ DTSSP (Pierce) and incubated for $2 \mathrm{~h}$ on ice. Coimmunoprecipitation was performed as described (Yeo and Whitman 2001). Samples were treated with $100 \mathrm{mM}$ DTT to cleave DTSSP prior to SDS-PAGE analysis. The following antibodies were used for immunoprecipitation and Western blot analysis: anti-Flag mouse monoclonal antibody (clone M2, Sigma), antiHA mouse monoclonal antibody (clone 16B12, Covance), anti-HA rabbit polyclonal antibody (Y-11, Santa Cruz Biotech), anti-c-Myc rabbit polyclonal antibody (A-14, Santa Cruz Biotech), and anti-c-Myc mouse monoclonal antibody (clone Ab-1, Oncogene). Proteins were visualized using the Super Signal West Pico/Femo Chemiluminescent Substrate system (Pierce).

\section{Acknowledgments}

We thank members of the Schier, Brivanlou, and Yelon laboratories for discussions; Amanda Carmany-Rampey and Will Talbot for comments on the manuscript; S. Zimmerman, T. Bruno, and N. Dillon for fish care; and C. Yeo, M. Whitman, and R. Harland for sharing of plasmids and protocols. S.K.C. was supported by NIH training grant T32HD07520. A.H.B. is supported by the NIH. A.F.S. is a Scholar of the McKnight Endowment Fund for Neuroscience, a Irma T. Hirschl Trust Career Scientist, an Established Investigator of the American Heart Association, and supported by grants from the NIH.

The publication costs of this article were defrayed in part by payment of page charges. This article must therefore be hereby marked "advertisement" in accordance with 18 USC section 1734 solely to indicate this fact.

\section{References}

Bamford, R.N., Roessler, E., Burdine, R.D., Saplakoglu, U., dela Cruz, J., Splitt, M., Goodship, J.A., Towbin, J., Bowers, P., Ferrero, G.B., et al. 2000. Loss-of-function mutations in the EGF-CFC gene CFC1 are associated with human left-right laterality defects. Nat. Genet. 26: 365-369.

Bianco, C., Adkins, H.B., Wechselberger, C., Seno, M., Normanno, N., De Luca, A., Sun, Y., Khan, N., Kenney, N., Ebert, A., et al. 2002. Cripto-1 activates nodal- and ALK4dependent and -independent signaling pathways in mammary epithelial cells. Mol. Cell. Biol. 22: 2586-2597. 
Brennan, J., Norris, D., and Robertson, E.J. 2002. Nodal activity in the node governs left-right asymmetry. Genes \& Dev. 16: $2339-2344$.

Conlon, F.L., Lyons, K.M., Takaesu, N., Barth, K.S., Kispert, A., Herrmann, B., and Robertson, E.J. 1994. A primary requirement for nodal in the formation and maintenance of the primitive streak in the mouse. Development 120: 19191928.

Ding, J., Yang, L., Yan, Y.T., Chen, A., Desai, N., WynshawBoris, A., and Shen, M.M. 1998. Cripto is required for correct orientation of the anterior-posterior axis in the mouse embryo. Nature 395: 702-707.

Feldman, B., Gates, M.A., Egan, E.S., Dougan, S.T., Rennebeck, G., Sirotkin, H.I., Schier, A.F., and Talbot, W.S. 1998. Zebrafish organizer development and germ-layer formation require nodal-related signals. Nature 395: 181-185.

Gaio, U., Schweickert, A., Fischer, A., Garratt, A.N., Muller, T., Ozcelik, C., Lankes, W., Strehle, M., Britsch, S., Blum, M., et al. 1999. A role of the cryptic gene in the correct establishment of the left- right axis. Curr. Biol. 9: 1339-1342.

Gritsman, K., Zhang, J., Cheng, S., Heckscher, E., Talbot, W.S., and Schier, A.F. 1999. The EGF-CFC protein one-eyed pinhead is essential for nodal signaling. Cell 97: 121-132.

Hata, A., Seoane, J., Lagna, G., Montalvo, E., Hemmati-Brivanlou, A., and Massague, J. 2000. OAZ uses distinct DNA- and protein-binding zinc fingers in separate BMP-Smad and Olf signaling pathways. Cell 100: 229-240.

Hemmati-Brivanlou, A. and Melton, D.A. 1992. A truncated activin receptor inhibits mesoderm induction and formation of axial structures in Xenopus embryos. Nature 359: 609614.

Hyatt, B.A. and Yost, H.J. 1998. The left-right coordinator: The role of $\mathrm{Vgl}$ in organizing left-right axis formation. Cell 93: $37-46$.

Hyatt, B.A., Lohr, J.L., and Yost, H.J. 1996. Initiation of vertebrate left-right axis formation by maternal $\mathrm{Vg} 1$. Nature 384: 62-65.

Kessler, D.S. and Melton, D.A. 1995. Induction of dorsal mesoderm by soluble, mature Vg1 protein. Development 121: 21552164.

Lee, M.A., Heasman, J., and Whitman, M. 2001. Timing of endogenous activin-like signals and regional specification of the Xenopus embryo. Development 128: 2939-2952.

Lowe, L.A., Yamada, S., and Kuehn, M.R. 2001. Genetic dissection of nodal function in patterning the mouse embryo. Development 128: 1831-1843.

Massague, J. and Chen, Y.G. 2000. Controlling TGF- $\beta$ signaling. Genes \& Dev. 14: 627-644.

Norris, D.P., Brennan, J., Bikoff, E.K., and Robertson, E.J. 2002. The Foxh1-dependent autoregulatory enhancer controls the level of Nodal signals in the mouse embryo. Development 129: 3455-3468.

Ramsdell, A.F. and Yost, H.J. 1999. Cardiac looping and the vertebrate left-right axis: Antagonism of left-sided Vg1 activity by a right-sided ALK2-dependent BMP pathway. Development 126: 5195-5205.

Rankin, C.T., Bunton, T., Lawler, A.M., and Lee, S.J. 2000. Regulation of left-right patterning in mice by growth/differentiation factor-1. Nat. Genet. 24: 262-265.

Reissmann, E., Jornvall, H., Blokzijl, A., Andersson, O., Chang, C., Minchiotti, G., Persico, M.G., Ibanez, C.F., and Brivanlou, A.H. 2001. The orphan receptor ALK7 and the Activin receptor ALK4 mediate signaling by Nodal proteins during vertebrate development. Genes \& Dev. 15: 2010-2022.

Sakuma, R., Ohnishi, Y-i,, Meno, C., Fujii, H., Juan, H., Takeuchi, J., Ogura, T., Li, E., Miyazono, K., and Hamada, H. 2002.
Inhibition of Nodal signalling by Lefty mediated through interaction with common receptors and efficient diffusion. Genes Cells 7: 401-412.

Salomon, D.S., Bianco, C., Ebert, A.D., Khan, N.I., De Santis, M., Normanno, N., Wechselberger, C., Seno, M., Williams, K., Sanicola, M., et al. 2000. The EGF-CFC family: Novel epidermal growth factor-related proteins in development and cancer. Endocr. Relat. Cancer 7: 199-226.

Sampath, K., Rubinstein, A.L., Cheng, A.M., Liang, J.O., Fekany, K., Solnica-Krezel, L., Korzh, V., Halpern, M.E., and Wright, C.V. 1998. Induction of the zebrafish ventral brain and floorplate requires cyclops/nodal signalling. Nature 395: 185-189.

Schier, A.F. and Shen, M.M. 2000. Nodal signalling in vertebrate development. Nature 403: 385-389.

Seleiro, E.A., Connolly, D.J., and Cooke, J. 1996. Early developmental expression and experimental axis determination by the chicken Vg1 gene. Curr. Biol. 6: 1476-1486.

Shen, M.M. and Schier, A.F. 2000. The EGF-CFC gene family in vertebrate development. Trends Genet. 16: 303-309.

Shen, M.M., Wang, H., and Leder, P. 1997. A differential display strategy identifies Cryptic, a novel EGF-related gene expressed in the axial and lateral mesoderm during mouse gastrulation. Development 124: 429-442.

Skromne, I. and Stern, C.D. 2002. A hierarchy of gene expression accompanying induction of the primitive streak by Vg1 in the chick embryo. Mech. Dev. 114: 115-118.

Thomsen, G.H. and Melton, D.A. 1993. Processed Vg1 protein is an axial mesoderm inducer in Xenopus. Cell 74: 433-441.

Wall, N.A., Craig, E.J., Labosky, P.A., and Kessler, D.S. 2000. Mesendoderm induction and reversal of left-right pattern by mouse Gdf1, a Vg1-related gene. Dev. Biol. 227: 495-509.

Weeks, D.L. and Melton, D.A. 1987. A maternal mRNA localized to the vegetal hemisphere in Xenopus eggs codes for a growth factor related to TGF- $\beta$. Cell 51: 861-867.

Whitman, M. 2001. Nodal signaling in early vertebrate embryos: Themes and variations. Dev. Cell 1: 605-617.

Yan, Y.T., Gritsman, K., Ding, J., Burdine, R.D., Corrales, J.D., Price, S.M., Talbot, W.S., Schier, A.F., and Shen, M.M. 1999. Conserved requirement for EGF-CFC genes in vertebrate left-right axis formation. Genes \& Dev. 13: 2527-2537.

Yan, Y.T., Liu, J.J., Luo, Y., E, C., Haltiwanger, R.S., Abate-Shen, C., and Shen, M.M. 2002. Dual roles of Cripto as a ligand and coreceptor in the nodal signaling pathway. Mol. Cell. Biol. 22: 4439-4449.

Yeo, C. and Whitman, M. 2001. Nodal signals to Smads through Cripto-dependent and Cripto-independent mechanisms. Mol. Cell 7: 949-957.

Zhang, J., Talbot, W.S., and Schier, A.F. 1998. Positional cloning identifies zebrafish one-eyed pinhead as a permissive EGFrelated ligand required during gastrulation. Cell 92: 241-251.

Zimmerman, L.B., De Jesus-Escobar, J.M., and Harland, R.M. 1996. The Spemann organizer signal noggin binds and inactivates bone morphogenetic protein 4. Cell 86: 599-606. 


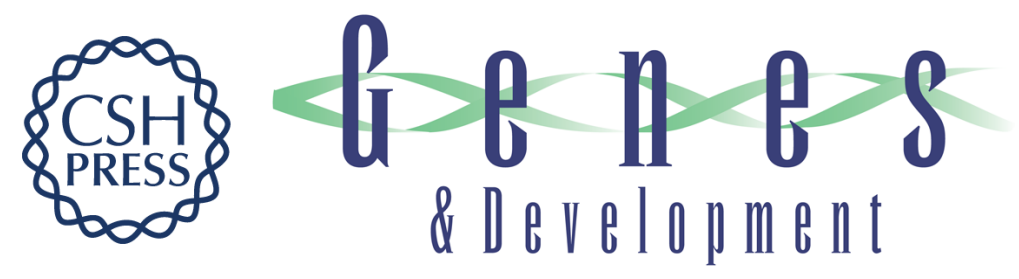

\section{EGF-CFC proteins are essential coreceptors for the TGF- $\beta$ signals Vg1 and GDF1}

Simon K. Cheng, Felix Olale, James T. Bennett, et al.

Genes Dev. 2003, 17:

Access the most recent version at doi:10.1101/gad.1041203

References This article cites 37 articles, 14 of which can be accessed free at: http://genesdev.cshlp.org/content/17/1/31.full.html\#ref-list-1

License

Email Alerting

Receive free email alerts when new articles cite this article - sign up in the box at the top Service right corner of the article or click here.

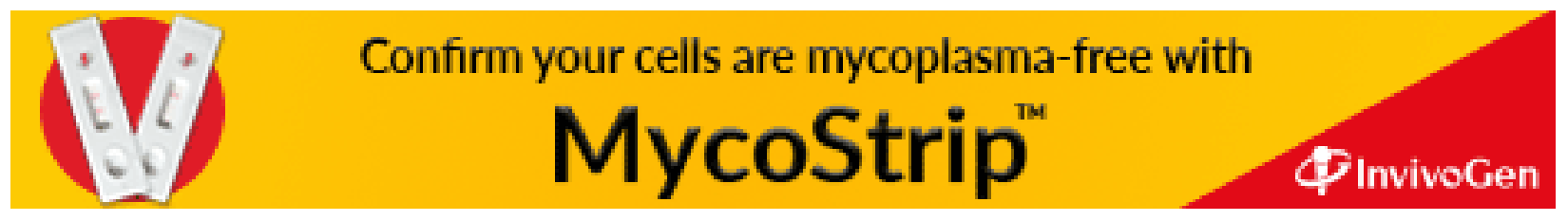

